\title{
A new exercise test for the assessment of heart failure: use of a self powered treadmill
}

\author{
J PARAMESHWAR, J-H E DAMBRINK, J SPARROW, C WRIGHT, A PARK, \\ J TWEED, P A POOLE-WILSON
}

\begin{abstract}
From the National Heart and Lung Institute and National Heart and Chest Hospitals, Dovehouse Street, London
\end{abstract}

SUMmaRY Time limited exercise on a self powered treadmill was evaluated as a method of assessing functional capacity in patients with mild heart failure. The characteristics of the treadmill were established by exercising 11 controls at three speeds and two inclinations and comparing oxygen consumption with that on a motorised treadmill under the same conditions. Oxygen consumption on the self powered treadmill at an equivalent speed and inclination was significantly higher because of the work needed to overcome the friction of the belt. Unlike a conventional treadmill, increasing the gradient on the self powered treadmill did not increase oxygen consumption. The distance walked in 12 minutes on the self powered treadmill was measured in eight patients with mild heart failure and ten controls. Maximal oxygen consumption was measured in the same group on a conventional treadmill by a mass spectrometer. There was a significant correlation between the distance walked and maximum oxygen consumption.

In patients with mild heart failure the distance travelled in 12 minutes on a self powered treadmill provides a practical, inexpensive, and sensitive method of assessing functional capacity.

The measurement of respiratory gas exchange during a maximal exercise test is established as an objective and reproducible method of assessing exercise capacity in patients with heart failure. ${ }^{1-3}$ The method is not widely available because the equipment is expensive and the procedure needs staff, training, and time. The end points are not entirely objective although knowledge of the maximum oxygen consumption and the respiratory ratio at peak exercise greatly ease the interpretation of an exercise test. Maximal exercise testing can be unpleasant for patients and worrying to doctors.

The six minute walking test was devised as an inexpensive and simple alternative method of assessing exercise capacity. ${ }^{45}$ The maximum distance that patients can walk along a flat corridor in six minutes at a self selected speed is measured. The distance walked in a given time, rather than the maximum level of exercise that can be achieved, may relate more closely to a patient's symptoms, be more relevant to limitations of everyday activities, and may be more

Requests for reprints to Dr J Parameshwar, National Heart Hospital, Westmoreland Street, London W1M 8BA.

Accepted for publication 24 January 1989 acceptable to patients.

The distance walked during a six minute walking test has been shown to discriminate better than measurement of maximal oxygen consumption between patients with severe chronic heart failure. In contrast the variation in the distance walked by patients with mild heart failure was small compared with the differences in maximum oxygen consumption. ${ }^{5}$ We report here a study of time limited exercise on a self powered treadmill as an alternative method that does not need a corridor. The objectives were threefold: firstly, to establish the characteristics of the treadmill in terms of oxygen consumption; secondly, to determine whether and how the distance walked related to maximum oxygen consumption; and thirdly, to measure oxygen consumption during the walking test.

\section{Patients and methods}

We studied 11 controls (mean age 32, range 25-44 years) and eight patients with mild or moderate heart failure (mean age 64, range 52-73 years). The controls were believed to be free of cardiac disease. The cause of heart failure was coronary artery disease in six patients and uncertain in two. The mean 
duration of heart failure was five years (range 2-13 years). All the patients were on a loop diuretic (mean dose of frusemide $55 \mathrm{mg}$ ). Four patients were taking amiloride. No patient was taking an angiotensin converting enzyme inhibitor. One patient was in atrial fibrillation and was receiving digoxin to control the heart rate. The mean maximum oxygen consumption of patients was $20.5 \mathrm{ml} / \mathrm{kg} / \mathrm{min}$. Exclusion criteria were evidence of myocardial ischaemia on exercise testing, significant pulmonary disease, or inability to exercise for any reason other than dyspnoea or fatigue. No patient had chest pain or any change on the electrocardiogram during or after exercise.

The study groups exercised on a Tunturi Jogger 2 self powered treadmill. This consists of a conveyer belt that slides over an underplate by the force of the subject walking on the belt. A liquid crystal display can show the distance travelled, the current speed, or the time elapsed, or will scan between these three. The distance walked was also recorded by a Trumeter surveyor's wheel attached to the belt of the treadmill. Both controls and patients practised on the treadmill before the study started. Most patients had performed the test at least five times before the study.

The oxygen consumption of 11 controls was measured at gradients of $4.4 \%$ and $11.1 \%\left(4^{\circ}\right.$ and $10^{\circ}$ respectively). Subjects walked at 3,5 , and $7 \mathrm{~km} / \mathrm{h}$. Each stage lasted three minutes, giving a total of nine minutes of continuous exercise. Similar measurements of oxygen consumption were made on a conventional motorised treadmill at a gradient of $11.1 \%$ and at the same three speeds.

Ten controls and the eight patients were asked to walk for 12 minutes on the Tunturi treadmill, set at a gradient of $6.7 \%\left(6^{\circ}\right)$. All were asked to walk the greatest distance possible at any speed they chose. The distance walked was recorded. Oxygen consumption and carbon dioxide production were monitored throughout the test.

We measured maximum oxygen consumption on a conventional treadmill (Bruce protocol) by an argon dilution technique and a mass spectrometer. ${ }^{16}$ The anaerobic threshold and extrapolated maximum consumption were obtained by computerised analysis with a curve fitting model. ${ }^{17}$

Results are expressed as mean (SD). Differences were analysed by analysis of variance and Student's $t$ test.

\section{Results}

COMPARISON BETWEEN GRADIENTS ON THE SELF POWERED TREADMILL

All the controls reported greater difficulty walking on the self powered treadmill at a gradient of $4.4 \%$ than at $11 \cdot 1 \%$. Only five completed nine minutes of exercise at both gradients; 10 completed six minutes $c$ at $4.4 \%$, and all 11 did so at $11 \cdot 1 \%$ (fig 1 ). At $4 \cdot 4 \% \vec{F}$ the mean oxygen consumption was $23.4(3.0), 32.8 \stackrel{5}{9}$ (3.9), and $41.1(3.5) \mathrm{ml} / \mathrm{kg} / \mathrm{min}$ at speeds of 3,5 , and $\bar{C}$ $7 \mathrm{~km} / \mathrm{h}$ respectively. At a gradient of $11 \cdot 1 \%$ the $\frac{\overline{\bar{O}}}{\mathrm{C}}$ corresponding values were $21 \cdot 3(2 \cdot 2), 30 \cdot 5(3 \cdot 7)$, and $\frac{\text { D }}{\sigma}$ $40.7(3.3) \mathrm{ml} / \mathrm{kg} / \mathrm{min}$ respectively. This difference $\mathbb{\Phi}$ was significant at $3 \mathrm{~km} / \mathrm{h}(\mathrm{p}<0.02)$ but not at higher ${ }_{\omega}$ speeds.

At the two lower speeds ( 3 and $5 \mathrm{~km} / \mathrm{h}$ ) and at $\mathrm{a} \stackrel{\circ}{\circ}$ slope of $4.4 \%$ the oxygen consumption correlated $\vec{\omega}$ inversely with weight of the controls $\left(r=-0.83\right.$, $\frac{\Omega}{P}$ $p<0.002$ and $r=0.75, p<0.01$ ). No correlations were apparent under any other conditions studied.

\section{COMPARISON BETWEEN TREADMILLS}

All the controls reported greater difficulty exercising on the self powered treadmill than on a conventional $?$ motorised one at comparable slopes and speeds. Ten completed nine minutes of exercise on the motorised treadmill. At a fixed gradient of $11.1 \%$ the mean oxygen consumption on the conventional treadmill $\infty$ was $16 \cdot 4(2 \cdot 0), 21 \cdot 9(2 \cdot 1)$, and $27 \cdot 1(2 \cdot 0) \mathrm{ml} / \mathrm{kg} / \mathrm{min}$ at 3 , 5 , and $7 \mathrm{~km} / \mathrm{h}$ respectively (fig 1 ). This was significantly less than the corresponding values on the self powered treadmill at all speeds $(\mathrm{p}<0.001)$.

\section{MAXIMAL OXYGEN CONSUMPTION}

Maximal oxygen consumption was limited by symp- $\overrightarrow{\vec{\rho}}$ toms in patients and controls. The mean maximal $\frac{9}{3}$ oxygen consumption was $40.9(11.3) \mathrm{ml} / \mathrm{kg} / \mathrm{min}$ in the controls and $20.5(4 \cdot 1) \mathrm{ml} / \mathrm{kg} / \mathrm{min}$ in the patients. The mean extrapolated maximal oxygen consumption $^{7}$ was $45 \cdot 1(13.1) \mathrm{ml} / \mathrm{kg} / \mathrm{min}$ and $26(5.7) \mathrm{ml} / \mathrm{kg} /$

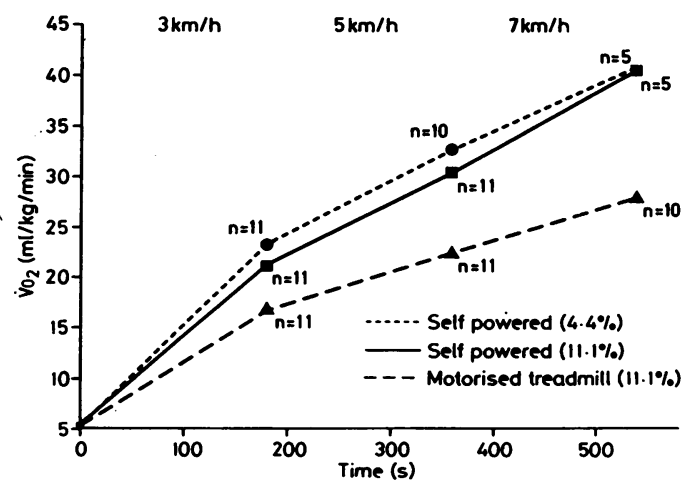

Fig 1 Oxygen consumption in controls after three minutes of exercise on the self powered treadmill at speeds of 3, 5, and $7 \mathrm{~km} / \mathrm{h}$. Not all subjects were able to exercise at the highest speed. For comparison the oxygen consumption on the motorised treadmill under similar conditons is shown. 


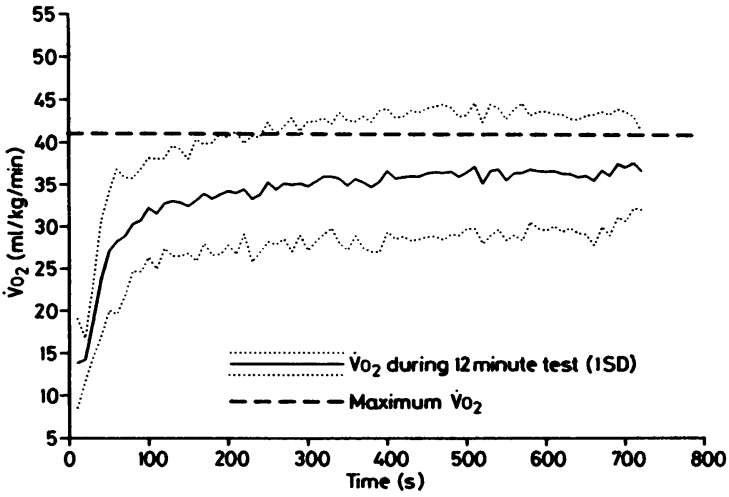

Fig 2 Oxygen consumption during the 12 minute walking test on the self powered treadmill in 11 controls. For comparison the maximum oxygen consumption of the group is also shown.

min respectively. The mean anaerobic threshold was $25(4.9) \mathrm{ml} / \mathrm{kg} / \mathrm{min}$ in the controls and $17.3(4.7) \mathrm{ml} /$ $\mathrm{kg} / \mathrm{min}$ in the patients. The mean respiratory quotient at peak exercise was 1.14 in controls and 1.06 in patients.

\section{TWELVE MINUTE WALKING TEST}

The mean distance walked in twelve minutes was $1052(195) \mathrm{m}$ in ten controls and $550(108) \mathrm{m}$ in the eight patients. The difference was significant $(p<0.001)$. During the test, oxygen consumption in patients and controls reached a plateau after approximately three minutes of exercise. This plateau value was $84 \%$ of the maximal oxygen consumption in the patients and $89 \%$ in controls (figs 2 and 3 ). The respiratory quotient at the end of the test was 1.03 in controls and 0.97 in patients. The respiratory quotient varied during the test and the

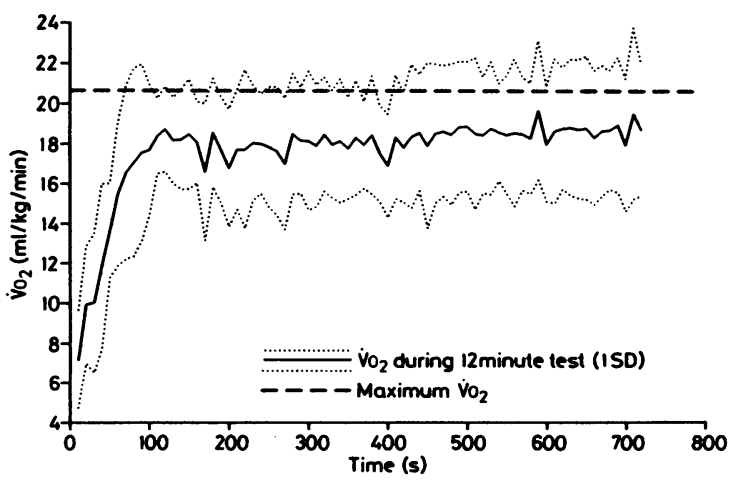

Fig 3 Oxygen consumption during the 12 minute walking test on the self powered treadmill in eight patients with mild heart failure. For comparison the maximum oxygen consumption of the patients is also shown.

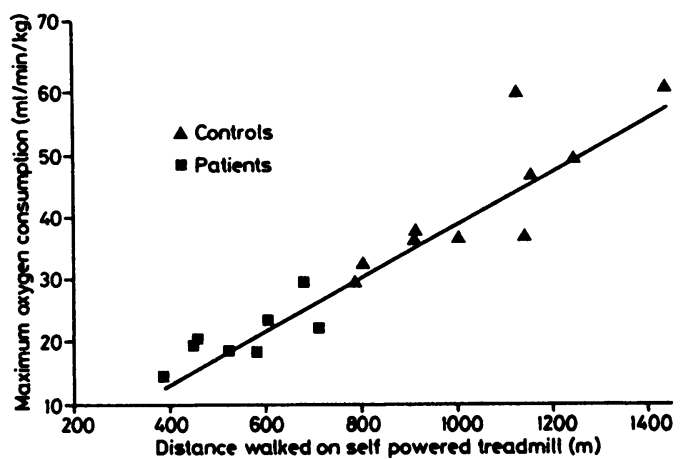

Fig 4 Maximum oxygen consumption determined from a standard exercise test and plotted against the distance walked in 12 minutes on the self powered treadmill in patients with mild heart failure and in controls $(r=0.85, p<0.005$ in controls, $r=0.75, p<0.05$ in patients, $r=0.94$, $p<0.0001$ for all persons).

value at the end of the test may be higher than during the test because of an attempt to walk faster in the last few moments of the test. The distance walked in 12 minutes correlated with maximal oxygen consumption in controls $(r=0.85, p<0.005)$ and in the patients $(r=0.75, p<0.05)$. When the results for the two groups were combined the correlation was highly significant $(r=0.94, p<0.0001)($ fig 4$)$. The extrapolated maximal oxygen consumption correlated with the distance walked in 12 minutes in the controls $(p<0.01)$ but not in the patients. When the results for the two groups were combined the correlation was significant $(r=0.89, p<0.001)$. The anaerobic threshold did not correlate with the distance walked in either group. There was also no correlation in either group between the distance walked in 12 minutes on the self powered treadmill and the exercise time on the conventional treadmill.

\section{Discussion}

Symptoms, often assessed by the New York Heart Association criteria for breathlessness, are a poor guide to the severity of heart failure ${ }^{89}$ and in recent years exercise testing has been widely used to classify patients with heart failure, to follow the progression of heart failure, and to assess the efficacy of new treatments. Despite this widespread use of exercise testing, particularly in clinical trials of drugs, ${ }^{10}$ there is no agreement on the most appropriate protocol or how to interpret the results. To circumvent the variability and subjectivity of the simple measurement of exercise duration, analysis of gas exchange during exercise and at peak exercise has become popular. Maximal oxygen consumption and 
anaerobic threshold are believed to provide more objective and reproducible end points. A knowledge of the respiratory quotient at peak exercise establishes whether the patient was truly limited by the cardiovascular system. ${ }^{11}{ }^{12}$ The equipment necessary to analyse respiratory gas exchange is expensive, however, and its use requires considerable technical skill. For these reasons the method is limited to a few centres. A more fundamental criticism of the method is that the relation between maximum exercise capacity and either symptoms or the ability to perform the common tasks of everyday life is not established.

The six minute walking test was put forward as an alternative test to assess patients with mild heart failure, which is cheap, reproducible, and easy to perform in most circumstances. The test measures the maximum distance a patient can walk at a self selected speed along a flat corridor in six minutes. Since the patient chooses the speed of walking the test may be more typical of normal activity than a conventional maximum exercise test. A major drawback to the test is that it does not appear to discriminate between patients with mild heart failure and normal subjects. ${ }^{5}$ Also a long corridor is required.

We modified the six minute walking test by extending the time to 12 minutes and by using a self powered treadmill rather than a corridor. The treadmill is convenient to use, allows better supervision of the patients, and is inexpensive and safe. The treadmill, however, is not simply a replacement for the corridor. The slope can be adjusted and considerable work is required to overcome the friction of the belt and inertia of the rollers. Thus it is a more strenuous test. The importance of these factors was not initially appreciated although we did expect that an increase of work on the treadmill might make the test more sensitive in patients with mild heart failure. The results in controls show that oxygen consumption was higher than on a motorised treadmill at all speeds by up to $15 \mathrm{ml} / \mathrm{kg} / \mathrm{min}$ (fig 1 ). The oxygen consumption did not increase on the self powered treadmill as the slope was increased from $4.4^{\circ}$ to $11 \cdot 1^{\circ}$. The reason for this observation is that overcoming the friction of the belt requires considerably less effort at the higher slope because body weight is more effective. The reduction in work required to overcome the friction of the belt must offset the greater oxygen consumption of walking up a steeper slope. At the lower slope and speeds body weight correlated inversely with oxygen consumption expressed per $\mathrm{kg}$ body weight. This was probably because heavier people were more effective in overcoming the friction of the belt under these conditions.

The mean maximum oxygen consumption of the patients was $20.5 \mathrm{ml} / \mathrm{kg} / \mathrm{min}$, indicating mild to moderate heart failure. ${ }^{1}$ There was a significan difference in the distance walked in twelve minutes: by the patients and controls. Although the controls? were not age matched it is unlikely that their youngero ages alone accorded for the large difference in theo distance walked. Maximum oxygen consumption in normal population of the same age as our patients is $33 \mathrm{ml} / \mathrm{kg} / \mathrm{min}^{13}$ compared with the value in oun controls of $40.9 \mathrm{ml} / \mathrm{kg} / \mathrm{min}$ and $20.5 \mathrm{ml} / \mathrm{kg} / \mathrm{min}$ in the patients. The distance walked correlated with the maximum oxygen consumption in patients, in con $\overrightarrow{\vec{\omega}}$ trols, and in the total study group. The relation $\omega$ seemed to be linear (fig 1). Patients walked approximately half the distance of controls. There is, $\sigma$ therefore, a substantial potential for a detectable $\vec{r}$ improvement in response to treatment.

The correlation between extrapolated maxima oxygen consumption ${ }^{7}$ and the distance walked in 129 minutes on a self powered treadmill was significantfor controls but not for patients. The anaerobic threshold was originally introduced as an objective measure of a person's exercise capacity. ${ }^{14}$ In the present study the anaerobic threshold did nofo correlate with the distance walked in the 12 minutio walking test. This is probably because of inaccuracies in calculating the anaerobic threshold. Indeed thes whole concept and existence of an anaerobic thres hold can be challenged. ${ }^{7}$ No correlation was foundo between exercise time on a conventional treadmile and the distance walked during the 12 minutee walking test. This finding is initially surprising bu? may reflect the many factors that determine exercise time on a motorised treadmill and account for differences between maximum oxygen consumption and exercise time. These include muscle mass, body weight, training effects, walking methods ${ }^{15}$ and mus $-{ }^{-}$ cle fibre type in different people. Another factor is the curvilinear relation between work performed an time on a motorised treadmill when a Bruce protoco $\bar{R}$ is followed.

The distance travelled during a time limited exer $\rightarrow$ cise test will depend on the ability of the subject to․ pace himself optimally. In general, subjects were ablo to pace themselves appropriately. Oxygen consump tion during the test reached a plateau in both controls and patients (figs 2 and 3). People in both groups took approximately three minutes to reach a steady state o oxygen consumption. There was a small increase of oxygen consumption in the last few seconds of the् test that was particularly noticeable in normal peo + ple. This represented their final effort to increase the distance walked as the end of the test approached Patients tended to walk at an oxygen consumption? nearer to their maximum than controls; the difference was not large (figs 2 and 3 ). 
The major difficulty in evaluating any new test for assessing patients with heart failure is that there is no absolute standard or measure of heart failure with which to compare the test. Haemodynamic variables at rest or exercise are known not to correlate with symptoms or exercise capacity. Measures of quality of life have the advantage of being a logical approach but they also reflect biases in the designer of the questionnaire, are subjective, and are difficult, if not impossible, to validate. This accounts for the interest in exercise testing to assess patients with heart failure. The test that we describe has important advantages over other exercise protocols. It is easy to perform, mimics normal activity, is cheap, presents a reasonable challenge to the patient, is acceptable to the patient, and has the sensitivity to detect improvement. The test seems particularly well suited to patients with mild or moderate heart failure but less practical in severe heart failure because of the effort needed to overcome the friction of the belt. None the less, the test may be useful in patients with severe heart failure because they can choose their own speed of walking.

We arbitrarily chose 12 minutes for the duration of the test believing that it was a suitable time over which to assess a patient without other factors such as loss of concentration contributing to the outcome. With hindsight we think that the test could have been shortened to nine minutes. A six minute test may be insufficient to establish a steady rate of exercise. The test may be suitable not only for patients with mild or moderate heart failure but also for patients with severe heart failure.

We thank Stuart Pharmaceuticals for the purchase of the Tunturi treadmill.

\section{References}

1 Lipkin DP, Perrins J, Poole-Wilson PA. Respiratory gas exchange in the assessment of patients with impaired ventricular function. Br Heart $J$ 1985; 54:321-8.

2 Weber KT, Kinasewitz GT, Janicki JS, Fishman AP. Oxygen utilisation and ventilation during exercise in patients with chronic cardiac failure. Circulation 1982;65:1213-23.

3 Wilson JR, Fink LI, Ferraro N, Dunkman WB, Jones RA. Use of maximal bicycle exercise testing with respiratory gas analysis to assess exercise performance in patients with congestive heart failure secondary to coronary artery disease or idiopathic dilated cardiomyopathy. Am J Cardiol 1986;58:601-6.

4 Guyatt GH, Sullivan MJ, Thompson PI, et al. The 6minute walk: a new measure of exercise capacity in patients with chronic heart failure. Can Med Assoc J 1985;132:919-23.

5 Lipkin DP, Scriven AJ, Crake T, Poole-Wilson PA. Six minute walking test for assessing exercise capacity in chronic heart failure. $\mathrm{Br}$ Med J 1986;292:653-5.

6 Davies N, Dennison DM. Measurement of metabolic gas exchange and minute volume by mass spectrometry alone. Respir Physiol 1979;36:261-7.

7 Buller NB, Poole-Wilson PA. Extrapolated maximum oxygen consumption: a new method for the objective analysis of respiratory gas exchange during exercise. Br Heart J 1988;59:212-7.

8 Lipkin DP. The role of exercise testing in chronic heart failure. Br Heart J 1987;58:559-66.

9 Weber KT, Janicki JS. Cardiopulmonary exercise for evaluation of chronic heart failure. Am J Cardiol 1985;55:22A-31A.

10 Lipkin DP, Poole-Wilson PA. Treatment of chronic heart failure: a review of recent trials. $\mathrm{Br} M e d \mathrm{~J}$ 1985;291:993-6.

11 Franciosa JA. Exercise testing in chronic congestive heart failure. Am J Cardiol 1984;53:1447-50.

12 Wasserman K, Whipp BJ, Koyal SN, Beaver WL. Anaerobic threshold and respiratory gas exchange during exercise. J Appl Physiol 1973;35:236-43.

13 Astrand P-O, Rodahl K. Textbook of work physiology. New York: McGraw-Hill, 1970.

14 Wasserman K, McIlroy MB. Detecting the threshold of anaerobic metabolism in cardiac patients during exercise. Am J Cardiol 1964;14:844-52.

15 Froelicher VF. Exercise testing and training: clinical applications. J Am Coll Cardiol 1983;1:114-25. 